Bull. Austral. Math. Soc.

VoL. 45 (1992) [195-200]

\title{
OSCILLATION OF NEUTRAL DELAY DIFFERENTIAL EQUATIONS
}

\section{Jianshe Yu, Zhicheng Wang and ChUanXi Qian}

Consider the following neutral delay differential equation

$$
\frac{d}{d t}[x(t)+P x(t-r)]+Q(t) x(t-\sigma)=0, \quad t \geqslant t_{0}
$$

where $P \in \mathbb{R}, \tau \in(0, \infty), \sigma \in[0, \infty)$ and $Q \in C\left[\left[t_{0}, \infty\right),[0, \infty)\right]$. We obtain a sufficient condition for the oscillation of all solutions of Equation $\left(^{*}\right)$ with $P=-1$, which does not require that

$$
\int_{t_{0}}^{\infty} Q(s) d s=\infty .
$$

But, for the cases $-1<P<0$ and $P<-1$, we show that $\left(^{* *}\right)$ is a necessary condition for the oscillation of all solutions of Equation $\left(^{*}\right)$. These new results solve some open problems in the literature.

\section{INTRODUCTION}

Consider the following neutral delay differential equation

$$
\frac{d}{d t}[x(t)+P x(t-\tau)]+Q(t) x(t-\sigma)=0, \quad t \geqslant t_{0}
$$

where

$$
P \in \mathbb{R}, \quad \tau \in(0, \infty), \quad \sigma \in[0, \infty) \text { and } Q \in C\left[\left[t_{0}, \infty\right),[0, \infty)\right]
$$

Recently, oscillation and asymptotic behaviours of Equation (1) have been investigated by several authors. (For a survey, see [2].) There is an interesting problem remaining. From all the known oscillation results for Equation (1) in the literature, it seems that

$$
\int_{t_{0}}^{\infty} Q(s) d s=\infty
$$

is an essential condition. Moreover, (3) is also a sufficient condition for the oscillation of all solutions of Equation (1) with $P=-1$, which has been established in [3]; see also [1]. Therefore, Chuanxi and Ladas posed the following question in [1].

Received 19 February 1991

Projects supported by the National Natural Science Foundation of the People's Republic of China.

Copyright Clearance Centre, Inc. Serial-fee code: 0004-9729/92 \$A2.00+0.00. 
Problem 1: Is condition (3) a necessary condition for the oscillation of all solutions of Equation (1) with $P=-1$ ?

In addition, Györi and Ladas recently put forward the following question in [2, Problem 6.12.10(a)].

Problem 2: In the case $-1 \leqslant P<0$, find sufficient conditions for the oscillation of all solutions of Equation (1) under the indicated restrictions on the function $Q$ and the delays $\tau$ and $\sigma$. Do not assume that (3) holds.

Our aim in this paper is to answer the above equations. We shall provide a sufficient condition which is weaker than (3) for the oscillation of all solutions of Equation (1) with $P=-1$. In addition, we shall show that if $-1<P<0$ (or $P<-1$ ) and (3) does not hold, then Equation (1) has a nonoscillatory solution and so (3) is indeed a necessary condition for the oscillation of all solutions of Equation (1) with $-1<P<0$ (or $P<-1$ ).

Let $t_{1} \geqslant t_{0}$ and let $\phi \in C\left[\left[t_{1}-\rho, t_{1}\right], \mathbb{R}\right]$, where $\rho=\max \{\tau, \sigma\}$. By a solution of (1) with initial function $\phi$ at $t_{1}$ we mean a function $x \in C\left[\left[t_{1}-\rho, \infty\right), \mathbb{R}\right]$ such that $x(t)=\phi(t)$ for $t \in\left[t_{1}-\rho, t_{1}\right], x(t)+P x(t-\tau)$ is continuously differentiable for $t \geqslant t_{1}$, and $x(t)$ satisfies (1) for all $t \geqslant t_{1}$.

As usual, a solution of (1) is called oscillatory if it has arbitrarily large zeros and nonoscillatory if it is eventually positive or eventually negative.

In the sequel, for convenience, we shall assume that inequalities about values of functions are satisfied eventually for all large $t$.

\section{Oscillation of Equation (1) With $P=-1$}

In this section, we study the oscillation of Equation (1) with $P=-1$. The following theorem is the main result.

TheOREM 1. Assume that (2) holds with $P=-1$. Suppose also

$$
\int_{t_{0}}^{\infty} s Q(s)\left(\int_{0}^{\infty} Q(t) d t\right) d s=\infty .
$$

Then every solution of Equation (1) oscillates.

ProOF: Since (3) implies that all the solutions of Equation (1) oscillate, it suffices to show that all solutions of Equation (1) oscillate in the case that

$$
\int_{t_{0}}^{\infty} Q(s) d s<\infty .
$$

Assume, for the sake of contradiction, that Equation (1) has an eventually positive solution $x(t)$. Then there exists $t_{1} \geqslant t_{0}$ such that

$$
x(t-\rho)>0 \text { for } t \geqslant t_{1}
$$


where $\rho=\max \{\tau, \sigma\}$. Set $y(t)=x(t)-x(t-\tau)$. Then

$$
y^{\prime}(t)=-Q(t) x(t-\sigma) \leqslant 0, \quad t \geqslant t_{1}
$$

which implies that $y(t)$ is nonincreasing for $t \geqslant t_{1}$. Therefore $y(t)$ is eventually negative or eventually positive.

First, we assume that $y(t)<0$ eventually. Since $y(t)$ is nonincreasing, there exists $\alpha>0$ and $T \geqslant t_{1}$ such that

$$
y(t)<-\alpha \text { for } t \geqslant T
$$

Therefore

$$
x(T)=y(T)+x(T-\tau)<-\alpha+x(T-\tau)
$$

and it follows that

$$
x(T+n \tau)<-(n+1) \alpha+x(T-\tau) \rightarrow-\infty \text { as } n \rightarrow \infty
$$

which contradicts (6). Thus, $y(t)$ cannot be eventually negative.

Next, we assume that $y(t)>0$ eventually. In this case, we have $x(t)>x(t-\tau)$. Hence, there exists $M>0$ and $T^{\prime} \geqslant t_{1}$ such that $x(t-\rho)>M$ for $t \geqslant T^{\prime}$. Then from (7), it follows that

$$
y^{\prime}(t) \leqslant-M Q(t) \text { for } t \geqslant T^{\prime}
$$

Hence

$$
y(t) \geqslant M \int_{t}^{\infty} Q(s) d s \text { for } t \geqslant T^{\prime}
$$

and so

$$
x(t) \geqslant M \int_{t}^{\infty} Q(s) d s+x(t-\tau) \text { for } t \geqslant T^{\prime}
$$

Let $T^{\prime}+n \tau \leqslant t \leqslant T^{\prime}+(n+1) \tau$. Then we have

(9) $x(t) \geqslant M \int_{t}^{\infty} Q(s) d s+M \int_{t-\tau}^{\infty} Q(s) d s+\ldots+M \int_{t-(n-1) \tau}^{\infty} Q(s) d s+x(t-n \tau)$

which, together with (7), yields

$$
y^{\prime}(t) \leqslant-n M Q(t) \int_{t}^{\infty} Q(s) d s \triangleq-H(t) .
$$

By noting that $t / n \rightarrow \tau$ as $t \rightarrow \infty$, we see that

$$
\frac{H(t)}{t Q(t) \int_{t}^{\infty} Q(s) d s}=\frac{n M}{t} \rightarrow \frac{M}{\tau} \text { as } t \rightarrow \infty .
$$


Clearly, (4) and (11) imply that

$$
\int_{t_{0}}^{\infty} H(s) d s=\infty
$$

Then (10) and (12) yield

$$
y(t) \rightarrow-\infty \text { as } t \rightarrow \infty
$$

which contradicts the hypotheses that $y(t)$ is eventually positive.

Therefore all the solutions of Equation (1) oscillate. The proof is complete.

REMARK 1. Clearly, (4) is weaker than (3). Hence, Theorem 1 is an improvement of the known result in [3] mentioned above and gives Problem 1 a negative answer.

EXAMPLE 1. Consider the following neutral delay differential equation

$$
\frac{d}{d t}[x(t)-x(t-\tau)]+\frac{1}{t^{\alpha}} x(t-\sigma)=0
$$

where $\alpha \in(1,3 / 2]$. It is easy to see that (4) holds. Then by Theorem 1 , every solution of Equation (13) oscillates. However, condition (3) is not satisfied.

3. Nonoscillation of Equation (1) With $-1<P<0$ or $P<-1$

In this section, we study the nonoscillation of Equation (1) with $-1<P<0$ or $P<-1$.

TheOREM 2. Assume that (2) holds with $-1<P<0$. Suppose also that

$$
\int_{t_{0}}^{\infty} Q(s) d s<\infty
$$

Then Equation (1) has a nonoscillatory solution.

Proof: Choose a positive number $T>t_{0}$ sufficiently large such that for $t \geqslant T$,

$$
t-\tau \geqslant t_{0}, \quad t-\sigma \geqslant t_{0} \text { and } \int_{t}^{\infty} Q(s) d s \leqslant \frac{1+P}{2} .
$$

Let $X$ be the set of all continuous and bounded functions on $\left[t_{0}, \infty\right)$ with the sup-norm. Then $X$ is a Banach space. Set

$$
A=\left\{x \in X: 1 \leqslant x(t) \leqslant 2 \text { for } t \geqslant t_{0}\right\} .
$$

Then $A$ is a bounded, closed and convex subset of $X$. Define a mapping $S: A \rightarrow X$ as

$$
(S x)(t)=\left\{\begin{array}{l}
1+P-P_{x}(t-\tau)+\int_{t}^{\infty} Q(s) x(s-\sigma) d s, \quad t \geqslant T \\
(S x)(T), \quad t_{0} \leqslant t \leqslant T
\end{array}\right.
$$


Clearly, $S$ is continuous. For every $x \in A$ and $t \geqslant T$ we see that

$$
(S x)(t) \leqslant 1+P-2 P+2(1+P) / 2=2
$$

and

$$
(S x)(t) \geqslant 1+P-P+0=1 .
$$

Hence, $1 \leqslant(S x)(t) \leqslant 2$ for $t \geqslant t_{0}$ and so $S A \subset A$.

Now we show that $S$ is a contraction mapping on $A$. In fact, for every $x_{1}, x_{2} \in A$ and $t \geqslant T$ we have

$$
\begin{aligned}
\left|\left(S x_{1}\right)(t)-\left(S x_{2}\right)(t)\right| & \leqslant|P|\left|x_{1}(t-\tau)-x_{2}(t-\tau)\right|+\int_{t}^{\infty} Q(s)\left|x_{1}(s-\sigma)-x_{2}(s-\sigma)\right| d s \\
& \leqslant\left\|x_{1}-x_{2}\right\|[-P+(1+P) / 2] \\
& =(1-P) / 2\left\|x_{1}-x_{2}\right\| .
\end{aligned}
$$

Then it follows that

$$
\begin{aligned}
\left\|S x_{1}-S x_{2}\right\| & =\sup _{t \geqslant t_{0}}\left|\left(S x_{1}\right)(t)-\left(S x_{2}\right)(t)\right| \\
& =\sup _{t \geqslant T}\left|\left(S x_{1}\right)(t)-\left(S x_{2}\right)(t)\right| \\
& \leqslant(1-P) / 2\left\|x_{1}-x_{2}\right\| .
\end{aligned}
$$

Since $(1-P) / 2<1$, we see that $S$ is a contraction. Then by the Banach Contraction principle, $S$ has a fixed point $x \in A$, that is, $S x=x$. Clearly, $x(t)$ is a positive solution of Equation (1) on [T, $\infty$ ) and so the proof is complete.

THEOREM 3. Assume that (2) holds with $P<-1$ and that (14) holds. Then Equation (1) has a nonoscillatory solution.

Proof: Let $T \geqslant t_{0}$ be sufficiently large such that

$$
t-\sigma \geqslant t_{0} \text { and } \int_{t+\tau}^{\infty} Q(s) d s \leqslant-(1+P) / 2 \text { for } t \geqslant T .
$$

Let $X$ be the set of all continuous and bounded functions on $\left[t_{0}, \infty\right)$ with the sup-norm. Set

$$
A=\left\{x \in X:-(1+P) / 2 \leqslant x(t) \leqslant-P \text { for } t \geqslant t_{0}\right\} .
$$

Then $A$ is a bounded, closed and convex subset of $X$. Define a mapping $S: A \rightarrow X$ as

$$
(S x)(t)=\left\{\begin{array}{l}
-(1+P)-\frac{1}{P} \geqslant x(t+\tau)+\frac{1}{P} \int_{t+\tau}^{\infty} Q(s) x(s-\sigma) d s, \quad t \geqslant T \\
(S x)(T), \quad t_{0} \leqslant t \leqslant T .
\end{array}\right.
$$


Then by an argument similar to that in the proof of Theorem 2, we see that $S A \subset A$ and for every $x_{1}, x_{2} \in A$

$$
\left\|S x_{1}-S x_{2}\right\| \leqslant\left(-\frac{1}{p}\right)\left(1-\frac{1+P}{2}\right)\left\|x_{1}-x_{2}\right\|=\frac{-1+P}{2 P}\left\|x_{1}-x_{2}\right\| .
$$

$S$ is a contraction since $0<(-1+P) /(2 P)<1$. Then by the Banach contraction principle, $S$ has a fixed point $x \in A$. Clearly, $x(t)$ is a positive solution of Equation (1) on $[T, \infty)$ and so the proof is complete.

REMARK 2. Clearly, Theorems 2 and 3 imply that (3) is a necessary condition for the oscillation of all solutions of Equation (1) with $-1<P<0$ or $P<-1$. Hence, Theorem 2 and Theorem 1 give Problem 2 a complete answer, that is, for the case that $-1<P<0$ we could not find any sufficient conditions, but for case that $P=-1$, we indeed can find some sufficient conditions for the oscillation of all solutions of Equation (1) without hypothesis (3).

\section{REFERENCES}

[1] Q. Chuanxi and G. Ladas, 'Oscillations of neutral differential equations with variable coefficients', Appl. Anal. 32 (1989), 215-228.

[2] I. Györi and G. Ladas, Oscillation theory of delay differential equations with applications (Oxford University Press, 1991).

[3] R.G. Koplatadze and T.A. Chanturia, 'On oscillatory and monotonic solutions of first-order differential equations with retarded arguments', Differentsial'nye Uravneniya 8 (1982), 1463-1465. (in Russian).

Department of Applied Mathematics

Hunan University

Changsha

Hunan 410082

Peoples Republic of China
Department of Mathematics

The University of Rhode Island

Kingston

Rhode Island 02881

United States of America 